\title{
DUAL SUPERCONDUCTORS AND SU(2) YANG-MILLS
}

\author{
Antti J. Niem* \\ Department of Theoretical Physics, Uppsala University, P.O. Box 803, S-75108, Uppsala, Sweden
}

(Dated: November 13, 2018)

\begin{abstract}
We propose that the $\mathrm{SU}(2)$ Yang-Mills theory can be interpreted as a two-band dual superconductor with an interband Josephson coupling. We discuss various consequences of this interpretation including electric flux quantization, confinement of vortices with fractional flux, and the possibility that a closed vortex loop exhibits exotic exchange statistics.
\end{abstract}

PACS numbers:

Color confinement remains one of the great mysteries of modern high energy physics 1]. Several approaches have been proposed to explain why and how a SU(N) Yang-Mills theory supports confining colored strings, including a variety of dual superconductor and central vortex models [2]. But until now the relevant dynamical string degrees of freedom have not been identified.

Recently it has been proposed [3] that the string variables in a Yang-Mills theory could relate to an appropriate decomposion of the gauge field. In the simplest case of $\mathrm{SU}(2)$ considered here, we start by separating $A_{\mu}^{a}(a=1,2,3)$ into its $\mathrm{U}(1)$ Cartan component $A_{\mu}^{3} \equiv A_{\mu}$ while the off-diagonal $A_{\mu}^{i}(i=1,2)$ we combine into the complex variable

$$
W_{\mu}^{ \pm}=A_{\mu}^{1} \pm i A_{\mu}^{2}
$$

We introduce the $\mathrm{U}(1)$ covariant derivatives

$$
D_{\mu}^{i j}=\delta^{i j} \partial_{\mu}-g \epsilon^{i j} A_{\mu} \quad \text { and } \quad D_{\mu}^{ \pm}=\partial_{\mu} \pm i g A_{\mu}
$$

and with $F_{\mu \nu}=\partial_{\mu} A_{\nu}-\partial_{\nu} A_{\mu}$ we expand the Yang-Mills Lagrangian as follows,

$$
\begin{aligned}
& -\frac{1}{4}\left(F_{\mu \nu}^{a}\right)^{2}+\frac{\xi}{2}\left(D_{\mu}^{i j} A_{\mu}^{j}\right)^{2}+\mathcal{L}_{F P}=-\frac{1}{4}\left(F_{\mu \nu}^{3}\right)^{2}-\frac{1}{4} F_{\mu \nu}^{+} F_{\mu \nu}^{-}+\frac{\xi}{2}\left(D_{\mu}^{i j} A_{\mu}^{j}\right)^{2}+\mathcal{L}_{F P} \\
= & -\frac{1}{4}\left(F_{\mu \nu}+G_{\mu \nu}\right)^{2}-\frac{1}{2} F_{\mu \nu} G_{\mu \nu}+\frac{1}{2}\left|D_{\mu}^{+} W_{\nu}\right|^{2}-\frac{1}{2}\left|D_{\mu}^{+} W_{\mu}\right|^{2}+\frac{\xi}{2}\left|D_{\mu}^{+} W_{\mu}\right|^{2}+\mathcal{P}^{\star} D_{+}^{2} \eta+\mathcal{P} D_{-}^{2} \eta^{\star} \\
& +\frac{g^{2}}{2}\left[\mathcal{P}^{\star} W_{\mu}^{+} W_{\mu}^{+} \eta+\mathcal{P} W_{\mu}^{+} W_{\mu}^{-} \eta^{\star}-\mathcal{P}^{\star} W_{\mu}^{+} W_{\mu}^{+} \eta^{\star}-\mathcal{P} W_{\mu}^{-} W_{\mu}^{-} \eta\right]
\end{aligned}
$$

Here we have explicitely displayed a gauge fixing and Faddeev-Popov ghost $(\mathcal{P}, \eta)$ [4] only for the off-diagonal $A_{\mu}^{1,2}$ components. The remaining Cartan U(1) gauge invariance can be fixed similarly. But we shall find that this U(1) invariance can also be eliminated directly, by casting the Lagrangian in a form that involves only manifestly U(1) invariant variables. In the following we shall mostly exclude the ghosts from explicit equations. Their inclusion has very little if any conceptual impact.

For the present purposes it is important to note that the sole effect of the fourth term in the r.h.s. of (II) is a redefinition of the gauge parameter $\xi \rightarrow \xi-1$ in the gauge fixing (fifth) term. Thus these terms can be combined. In particular, in the $\xi \rightarrow \infty$ limit the fourth term becomes entirely immaterial. This limit yields the Landau version of the maximal abelian gauge

$$
\left(\partial_{\mu}+i g A_{\mu}\right) W_{\mu}=0
$$

which we shall assume has been imposed. We note that this gauge condition appears as the variational equation when one locates the extrema of the functional

$$
\int \rho^{2}=\int\left(\rho_{1}^{2}+\rho_{2}^{2}\right)=\int\left[\left(A_{\mu}^{1}\right)^{2}+\left(A_{\mu}^{2}\right)^{2}\right]=\int W_{\mu} W_{\mu}^{*}
$$

with respect to the full $\mathrm{SU}(2)$ gauge transformations. Thus, even though the field $\rho$ is in general a gauge dependent quantity, its extremum values with respect to gauge transformations are manifestly gauge independent when the theory is subject to the Landau version of maximal abelian gauge. Recent numerical investigations indicate that in strong coupling regime the extrema of $\rho$ are nonvanishing [5]

The antisymmetric tensor

$$
G_{\mu \nu}=(i g / 2)\left(W_{\mu} W_{\nu}^{\star}-W_{\nu} W_{\mu}^{\star}\right)
$$


modifies the Cartan part of the Lagrangian according to

$$
F_{\mu \nu}^{2} \rightarrow\left(F_{\mu \nu}^{3}\right)^{2}=\left(F_{\mu \nu}+G_{\mu \nu}\right)^{2}+2 G_{\mu \nu} F_{\mu \nu}
$$

Dirac proposed in [6] that this is in essence the way how Maxwellian electrodynamics should be improved to accout for both electric and magnetic sources, he identified $G_{\mu \nu}$ as a string tensor, the source term for the magnetic four-current. Here we reconcile ourselves with the customary terminology to describe confinement in magnetic dual variables [2]. Consequently we interpret $F_{\mu \nu}$ as a closed dual field strength tensor, and view

$$
E_{i}=-G_{o i} \quad \text { and } \quad H_{i}=-1 / 2 \epsilon_{i j k} G_{j k}
$$

as the electric and magnetic components of the (non-closed electric) string tensor $G_{\mu \nu}$, in a dual version of Dirac's electrodynamics.

We now proceed to the third term on the r.h.s. of (1), the sole remaining term with no ghost contributions. We follow [3] and introduce a complex vector field $\mathbf{e}_{\mu}$ with

$$
\mathbf{e}_{\mu} \mathbf{e}_{\mu}=0 \quad \text { and } \quad \mathbf{e}_{\mu} \mathbf{e}_{\mu}^{*}=1
$$

We also introduce two complex scalar fields $\psi_{1}$ and $\psi_{2}$, and decompose the off-diagonal $W_{\mu}=A_{\mu}^{1}+i A_{\mu}^{2}$ into $\underline{3}$ ]

$$
W_{\mu}=\psi_{1} \mathbf{e}_{\mu}+\psi_{2}^{\star} \mathbf{e}_{\mu}^{\star}
$$

When we substitute this in the third term of (1) we get

$$
\left|\left(\partial_{\mu}+i g A_{\mu}^{3}\right) W_{\nu}\right|^{2}=\left|\left(D_{\mu}^{+}+i \Gamma_{\mu}\right) \psi_{1}\right|^{2}+\left|\left(D_{\mu}^{-}+i \Gamma_{\mu}\right) \psi_{2}\right|^{2}+\rho^{2}\left|\left(\partial_{\mu}-i \Gamma_{\mu}\right) \mathbf{e}_{\nu}\right|^{2}+\psi_{1} \psi_{2}\left(\partial_{\mu} \mathbf{e}_{\nu}\right)^{2}+\psi_{1}^{\star} \psi_{2}^{\star}\left(\partial_{\mu} \mathbf{e}_{\nu}^{\star}\right)^{2}
$$

where $\rho$ is defined by (2) and

$$
\Gamma_{\mu}=i \mathbf{e}_{\nu} \partial_{\mu} \mathbf{e}_{\nu}^{*}
$$

is a (composite) gauge field for the internal $\mathrm{U}(1)$ rotation

$$
\psi_{1,2} \rightarrow e^{i \alpha} \psi_{1,2} \quad \text { and } \quad \mathbf{e}_{\mu} \rightarrow e^{-i \alpha} \mathbf{e}_{\mu}
$$

which leaves the decomposition (44) intact [3].

Here it is interesting to also spell out the four last ghost interaction terms on the r.h.s. of (11). We define the ghost number operator

$$
\hat{N}=\mathcal{P}^{\star} \eta+\mathcal{P} \eta^{\star}
$$

and combine these terms into

$$
\frac{g^{2}}{2} \rho^{2} \hat{N}-g^{2} \psi_{1} \psi_{2}^{\star} \mathcal{P}^{\star} \eta^{\star}-g^{2} \psi_{1}^{\star} \psi_{2} \mathcal{P} \eta
$$

(Note that $\rho$ gives an effective mass for the ghosts.) The r.h.s. of (5) and (6) admit a direct interpretation in terms of the Landau-Ginzburg free energy of a two-gap superconductor, employed to describe high- $T_{c}$ materials [], [8] such as the widely studied $\mathrm{MgB}_{2}[9]$. Indeed, the first two terms in the r.h.s. of (5) are conventional kinetic terms for two oppositely charged Cooper pairs, except that now these Cooper pairs are also minimally coupled to the additional $\mathrm{U}(1)$ gauge field $\Gamma_{\mu}$ so that we have a local $\mathrm{U}_{\mathrm{A}}(1) \times \mathrm{U}_{\Gamma}(1)$ symmetry. This additional gauge field can be viewed as a Josephson current which originates in a gauged nonlinear sigma-model, in the present case with a $\mathrm{O}(3,1)$ structure, which is defined by the third term in the r.h.s. of (5). In a condensed matter context such a term could model fluctuations in the underlying material lattice structure. Finally, the last two terms on the r.h.s. of (5) together with the last two terms in (6) have the standard form of interband Josephson transition terms between the two Cooper pair condensates [10]. When we group the condensates into multiplets in two alternative ways, with the $\mathrm{U}_{\mathrm{A}}(1)$ charge of $\left(\psi_{1}, \psi_{2}^{\star}\right)$ opposite to that of $\left(\psi_{1}^{\star}, \psi_{2}\right)$, and the $\mathrm{U}_{\Gamma}(1)$ charge of $\left(\psi_{1}, \psi_{2}\right)$ opposite to that of $\left(\psi_{1}^{\star}, \psi_{2}^{\star}\right)$, we find that the last two terms in (5) are interband Josephson transition terms between the $\mathrm{U}_{\mathrm{A}}(1)$ multiplets, while the last two terms in (66) describe interband transitions between the $\mathrm{U}_{\Gamma}(1)$ multiplets.

We are particularly interested in vortex-like configurations in (3), (5), since these are the natural candidates for describing confining strings. For this we define

$$
\psi_{i}=\rho_{i} \exp \left(i \theta_{i}\right) \quad(i=1,2)
$$


where according to (44), the $\rho_{1,2}$ are the same fields as those in (2). We introduce the supercurrent

$$
\begin{aligned}
J_{\mu}= & g A_{\mu}+\frac{i}{2\left(\rho^{2}+\hat{N}\right)}\left(W_{\nu} \partial_{\mu} W_{\nu}^{\star}-\partial_{\mu} W_{\nu} W_{\nu}^{\star}-\mathcal{P}^{\star} \partial_{\mu} \eta+\partial_{\mu} \mathcal{P}^{\star} \eta+\mathcal{P} \partial_{\mu} \eta^{\star}-\partial_{\mu} \mathcal{P} \eta^{\star}\right) \\
& \rightarrow g A_{\mu}+\frac{\rho_{1}^{2}}{\rho^{2}}\left(\partial_{\mu} \theta_{1}+\Gamma_{\mu}\right)-\frac{\rho_{2}^{2}}{\rho^{2}}\left(\partial_{\mu} \theta_{2}+\Gamma_{\mu}\right)
\end{aligned}
$$

where in the last step we have removed all ghost dependence, since the only role of ghosts is to BRST-improve the current. We note that $J_{\mu}$ is gauge invariant both under $\mathrm{U}_{\mathrm{A}}(1)$ and $\mathrm{U}_{\Gamma}(1)$. We also introduce the three component unit vector

$$
\mathbf{t}=\frac{1}{\rho^{2}}\left(\begin{array}{l}
\psi_{1}^{\star} \\
\psi_{2}
\end{array}\right) \vec{\sigma}\left(\begin{array}{c}
\psi_{1} \\
\psi_{2}^{\star}
\end{array}\right)=\frac{1}{\rho^{2}}\left(\begin{array}{c}
2 \rho_{1} \rho_{2} \cos \left(\theta_{1}+\theta_{2}\right) \\
-2 \rho_{1} \rho_{2} \sin \left(\theta_{1}+\theta_{2}\right) \\
\rho_{1}^{2}-\rho_{2}^{2}
\end{array}\right)
$$

which is invariant under the Cartan $U_{A}(1)$. By defining

$$
\left(\nabla_{\mu} \mathbf{t}\right)_{k}=\left(\delta_{k l} \partial_{\mu}-2 \epsilon_{k l 3} \Gamma_{\mu}\right) t_{l}
$$

we can then represent (the $\xi \rightarrow \infty$ limit of) the entire SU(2) Yang-Mills Lagrangian (1) in terms of Cartan $U_{A}(1)$ invariant variables 7] (we do now write ghosts explicitely): For the first two terms in the r.h.s. of (11) which correspond to Dirac's extension of (dual) electromagnetism we get

$$
\begin{gathered}
\left(F_{\mu \nu}+G_{\mu \nu}\right)^{2}+2 F_{\mu \nu} G_{\mu \nu}=H_{\mu \nu}^{2}+4 i \rho^{2} t_{3}\left(\mathbf{e}_{\mu} \mathbf{e}_{\nu}^{\star}-\mathbf{e}_{\nu} \mathbf{e}_{\mu}^{\star}\right) H_{\mu \nu}+\rho^{4} t_{3}^{2} \\
H_{\mu \nu}=\partial_{\mu} J_{\nu}-\partial_{\nu} J_{\mu}+\frac{1}{2} t_{3}\left(\partial_{\mu} \Gamma_{\nu}-\partial_{\nu} \Gamma_{\mu}\right)+\frac{1}{4} \epsilon_{a b c} t_{a} \nabla_{\mu} t_{b} \nabla_{\nu} t_{c}
\end{gathered}
$$

and the third term in the r.h.s. of (10) gives; see (5)

$$
\left|\left(\partial_{\mu}+i g A_{\mu}^{3}\right) W_{\nu}\right|^{2}=\left(\partial_{\mu} \rho\right)^{2}+\frac{1}{4}\left(\nabla_{\mu} \mathbf{t}\right)^{2}+4 \rho^{2} J_{\mu}^{2}+\rho^{2}\left|\left(\partial_{\mu}-i \Gamma_{\mu}\right) \mathbf{e}_{\nu}\right|^{2}+\frac{\rho^{2}}{2}\left[t_{+}\left(\partial_{\mu} \mathbf{e}_{\nu}\right)^{2}+t_{-}\left(\partial_{\mu} \mathbf{e}_{\nu}^{\star}\right)^{2}\right]
$$

where $t_{ \pm}=t_{1} \pm i t_{2}$.

In the case of superconducting materials one often implements the London limit, which assumes that $\rho_{1}$ ad $\rho_{2}$ are non-vanishing constants. In a superconductor they describe the densities of the Cooper pairs, and under appropriate conditions the London limit is a reasonable approximation. But in the present case these fields relate to extrema of (2) under gauge transformations, and the $\mathrm{SU}(2)$ gauge invariance of the London limit must be justified separately, for example by numerical investigations [5]. Consequently here the London limit is solely an analytically tractable simplification of (9), (11).

For asymptotically constant and non-vanishing densities $\rho_{1}$ and $\rho_{2}$, the abelian two-condensate Higgs model supports line vortices [11]. We wish to call them egorons, and we assume that they are not removed by our additional terms. These egorons are then natural candidates for describing confining strings, and we now analyze their properties:

We compute the (electric) flux of an egoron as follows: In the London limit the supercurrent $J_{\mu}$ describes a massive degree of freedom, which means that we have a Meissner effect with inverse London penetration length $\propto \rho$. We integrate $J_{\mu}$ along a closed path which encircles the egoron at a distance much larger than the London penetration length. On this path $J_{\mu}$ then vanishes, and we get from (17) for the flux

$$
\Phi=\oint A=\frac{\rho_{2}^{2}}{g \rho^{2}} \oint \partial_{\mu} \theta_{2}-\frac{\rho_{1}^{2}}{g \rho^{2}} \oint \partial_{\mu} \theta_{1}+\frac{\rho_{2}^{2}-\rho_{1}^{2}}{g \rho^{2}} \oint \Gamma=\frac{2 \pi}{g \rho^{2}}\left(\rho_{2}^{2} N_{2}-\rho_{1}^{2} N_{1}\right)-\frac{t_{3}}{g} \oint \Gamma
$$

where $\Delta \theta_{1,2}=2 \pi N_{1,2}$ (with $N_{1,2}$ integers) are the angular increments in the phases of $\psi_{1,2}$ when we encircle the $\left(N_{1}, N_{2}\right)$ egoron once.

Notice that in the absence of the Josephson contribution the flux appears to have arbitrary values, depending on the relative values of the asymptotic densities $\rho_{1}$ and $\rho_{2}$ [8]. This is in a stark contrast with the quantization of magnetic flux of the Abrikosov vortex in the single-component abelian Higgs model. In order to further study the flux quantization properties of egorons we rewrite the first two terms in the r.h.s. of (5) as

$$
\left|\left(D_{\mu}^{+}+i \Gamma_{\mu}\right) \psi_{1}\right|^{2}+\left|\left(D_{\mu}^{-}+i \Gamma_{\mu}\right) \psi_{2}\right|^{2}=\left(\partial_{\mu} \rho_{1}\right)^{2}+\left(\partial_{\mu} \rho_{2}\right)^{2}+\rho_{1}^{2}\left(g A_{\mu}+\Gamma_{\mu}+\partial_{\mu} \theta_{1}\right)^{2}+\rho^{2}\left(g A_{\mu}-\Gamma_{\mu}-\partial_{\mu} \theta_{2}\right)^{2}
$$


In the London limit with both $\rho_{1}$ and $\rho_{2}$ non-vanishing, the two supercurrents in (13)

$$
j_{\mu}^{1,2}=g A_{\mu} \pm\left(\Gamma_{\mu}+\partial_{\mu} \theta_{1,2}\right)
$$

do not describe independent field degrees of freedom, but are nevertheless massive composites that exhibit the Meissner effect. Consequently we can compute the (electric) flux of a linear $\left(N_{1}, N_{2}\right)$ egoron by integrating both (14) over a closed path which encircles the egoron at a distance much larger than the inverse London penetration lengths $\sim \rho_{1,2}$. This gives

$$
\Phi=\oint A=\frac{\pi}{g}\left(N_{2}-N_{1}\right) \quad \& \quad \oint \Gamma=-\pi\left(N_{1}+N_{2}\right)
$$

For consistency we note that when we substitute this in (12) we recover (15).

(A similar computation in the ghost sector with nonvanishing ghost number also leads to $\Phi$ in (15), but with $N_{1}$ and $N_{2}$ now referring to the phase increments in the ghost fields.)

The result (15) reveals that in the presence of an interband Josephson flow the flux of an egoron becomes quantized. However, for the simplest egorons with $\left(N_{1}, N_{2}\right)=( \pm 1,0)$ or $(0, \pm 1)$ we still obtain a flux quantization in half units of an Abrikosov vortex. But for an egoron with finite energy per unit length there is also an interband Josephson flow carried by $\Gamma_{\mu}$. Whenever this interband flow vanishes $\left(\Gamma_{\mu}=0\right)$, for finite energy per unit length we must have $N_{1}=-N_{2}=-N$ so that the (dual) flux $\Phi$ of an egoron acquires a quantization in integer units of the Abrikosov vortex, $\Phi=2 \pi / g N$.

We observe that in the absence of any interband Josephson coupling the present considerations lead to a curious (algebraic) confinement of egorons: If we set $\Gamma_{\mu} \equiv 0$ we conclude from (13) that an isolated $(0, \pm 1)$ or $( \pm 1,0)$ egoron has a logarithmically divergent energy per unit length, while (12) implies that its (electric) flux is a priori an arbitrary fraction of the Abrikosov flux. But a neutral bound state egoron of the form $( \pm 1, \mp 1)$ has a finite energy, and its flux is quantized in integer units of the Abrikosov vortex.

We now wish to bend a finite length linear egoron into a toroidal ring. But before joining the ends we twist the egoron once around its core. The closed ring then acquires a non-trivial self-linkage, and numerical simulations indicate that such knotted egorons can be stable, at least if we account for a definite, possibly radiatively induced correction term [12]. The structure of a closed knotted egoron is best described in terms of the unit vector $\mathbf{t}$ in (8). For a static finite energy egoron it defines a mapping from the compactified $R^{3} \sim S^{3} \rightarrow S^{2}$ and $\pi_{3}\left(S^{2}\right) \sim Z$ computes the egorons self-linking number; We refer to [13], 12] for details. The asymptotic large distance vacuum value of $\mathbf{t}$ is determined by minimizing the Landau-Ginzburg free energy, for example the last term of (9) in soliture would identify the asymptotic value of $\mathbf{t}$ to be the circle $t_{3}=0$ corresponding to equal asymptotic densities $\rho_{1}=\rho_{2}$. But in general $t_{3} \neq \pm 1$, as we only expect that both asymptotic densities $\rho_{1}$ and $\rho_{2}$ are non-vanishing [5].

A fixed asymptotic value $t_{3} \neq \pm 1$ defines a circle on the target $S^{2}$ and leaves us with a global U(1) invariance in the phase of $t_{ \pm}$. In a confining theory there are no massless modes, and consequently this global invariance must become broken. This is achieved by the interband Josephson couplings in (5), (6), or alternatively we simply combine the phase of $t_{ \pm}$with $\Gamma_{\mu}$ to form a massive composite with $\mathbf{e}_{\mu}$.

A massive $t_{ \pm}$identifies the vacuum with a definite point $\mathbf{t}_{0} \in S^{2}$, which we assume is different from the poles at $t_{3}= \pm 1$. The center of the egoron corresponds to the preimage of the antipodal $-\mathbf{t}_{0}$. This implies that the egoron can be viewed as a double-stranded composite of two mutually linked closed $\left(N_{1}, 0\right)$ and $\left(0, N_{2}\right)$ constituent egorons in the fields $\psi_{1}$ and $\psi_{2}$, respectively. The core of $\left(N_{1}, 0\right)$ is the preimage of the north-pole $t_{3}=+1$ where $\rho_{2}$ vanishes, and the core of $\left(0, N_{2}\right)$ is the preimage of the south-pole $t_{3}=-1$ where $\rho_{1}=0$. The relative linking number of these two closed constituent egorons coincides with the self-linking number of the knotted composite egoron. This double-stranded structure of egorons has also been observed in numerical simulations [11]; see [14] for a detailed analysis.

The two closed $\left(N_{1}, 0\right)$ and $\left(0, N_{2}\right)$ constituent egorons can be pulled through each other, provided we do not cross or otherwise alter their relative linking number: They can not break and rejoin, become split, or cross each other without the self-linkage structure of the composite egoron becoming destructed. This non-destructibility is direct consequence of the fact that their cores are the embeddings of two different $S^{1} \in R^{3}$, corresponding to the preimages of $t_{3}= \pm 1$, respectively. We now argue that as a consequence the exchange statistics of the two constituent egorons relates to the exchange statistics of anyons on a plane [15]. Indeed, the exchange of $N$ pointlike plane particles which leads to Artin's braid group of $N$ objects, is often pictured in terms of vertical strands that describe the time evolution of the particles, with each strand corresponding to the world-line of a particle 15. Artin's braid group is generated by elementary moves that braid these strands around each other in a definite manner. This pictorial representation relates directly to the linking of (non-destructible) loops in three dimensions. For this, simply draw the strands as 
lines on the surface of a cylinder and identify the initial and final points of the individual strands. The world-lines of the particles now become closed loops on the surface of the cylinder, and the nontrivial braiding translates to the nontrivial linking of the loops. But such loops are special cases of general closed loops embedded in $R^{3}$, now wrapped around the surface of the cylinder. This suggests that more generally, there must be a relation between the braiding of world-lines and the linking of closed loops in $R^{3}$. Indeed, one can show that Artin's braid group appears as a subgroup of motions when we exchange (non-destructible) three dimensional closed loops [16]. It is generated by a motion which corresponds to the pulling of two constituent egorons through each other without crossing or otherwise changing their relative linking number. This suggests that a knotted $\left(N_{1}, N_{2}\right)$ egoron exhibits internal anyonic exchange statistics.

Finally, we recall that the role of ghosts is to eliminate the unphysical gauge degrees of freedom from the YangMills action. For a fully gauge fixed theory we need to complete (1) by two additional ghosts, which cancel the two unphysical polarizations in the abelian $A_{\mu}^{3}$. The four ghosts displayed in (11) remove four unphysical field degrees of freedom among the eight which are represented by the variables $\psi_{1,2}$ and $\mathbf{e}_{\mu}$. We propose that this entirely removes $\mathbf{e}_{\mu}$, leaving the four $\psi_{1,2}$. From (3) and (5) we then conclude that the Yang-Mills theory must be intimately related to the abelian two-condensate superconductor with Lagrangian of the form

$$
-\frac{1}{4} F_{\mu \nu}^{2}+\left|\left(\partial_{\mu}+i g A_{\mu}\right) \psi_{1}\right|^{2}+\left|\left(\partial_{\mu}-i g A_{\mu}\right) \psi_{2}\right|^{2}-\lambda\left(\left|\psi_{1}\right|^{2}-\left|\psi_{2}\right|^{2}\right)^{2}+\gamma\left(\psi_{1}^{\star} \psi_{2}+\psi_{2}^{\star} \psi_{1}\right)
$$

This is indeed a manifestly unitary theory for six physical field degrees of freedom, the two transverse components of the U(1) gauge field together with the four scalars. The $\lambda$ and $\gamma$ are determined dynamically, by the underlying YangMills theory. Furthermore, if we compute the one-loop Yang-Mills $\beta$-function from (16), despite it being an abelian theory we nevertheless obtain the correct $\mathrm{SU}(2)$ result provided we renormalize (16) by respecting the underlying $\mathrm{SU}(2)$ gauge structure. This can be confirmed either by a comparison with the results in [4], or by a direct evaluation of the one-loop scalar field effective potential in (16) and then scaling the fields in a manner which is consistent with the underlying $\mathrm{SU}(2)$ structure; as detailed in [17] this leads to the correct $\mathrm{SU}(2) \beta$-function, further supporting our proposal that (16) is the right superconductor model for $\mathrm{SU}(2)$ Yang-Mills theory.

In conclusion, we have argued that SU(2) Yang-Mills theory can be mapped into a two-gap superconductor with interband Josephson couplings. This model supports line vortices, which are then candidates for confining strings. The vortices have also curious properties including a priori fractional flux, algebraic confinement, and the possibility that closed vortex rings exhibit exotic internal statistics. Finally, we argued that the superconductor model leads to the correct Yang-Mills $\beta$-function.

We thank E. Babaev, T. Ekholm, L. Faddeev and O. Viro for discussion, and ICTP at Trieste for hospitality. This work is supported by grant VR-2003-3466. 
* Electronic address: niemi@teorfys.uu.se

† URL: http://www.teorfys.uu.se/people/antti

[1] Color confinement is one of the seven Millenium Problems; see http://www.claymath.org

[2] for recent review, see G. Ripka, Dual Superconductor Models of Color Confinement (Lecture Notes in Physics 639, Springer Verlag 2004)

[3] L.D. Faddeev and A.J. Niemi, Phys. Lett. B525 (2002) 195; T.A. Bolokhov and L.D. Faddeev, Theor. Math. Phys. (to appear)

[4] H. Min, T. Lee and P.Y. Pac, Phys. Rev. D32 (1985) 440

[5] F.V. Gubarev, L. Stodolski and V.I. Zakharov, Phys. Rev. Lett. 86 (2001) 2220; L. Stodolsky, P. van Baal and V.I. Zakharov, Phys. Lett. B552 (2003) 214

[6] P. Dirac, Phys. Rev. bf 74 (1948) 817

[7] E. Babaev, L. Faddeev and A.J. Niemi, Phys. Rev B65 (2002) 100512

[8] E. Babaev, Phys. Rev. Lett. 89 (2002) 067001

[9] F. Bouquet et.al. Phys. Rev. lett. 87 (2001) 047001; Amy Y. Liu et.al. Phys. Rev. Lett. 87 087005; P. Szabo et.al. Phys. Rev. Lett. 87 (2001) 137005

[10] A.J. Leggett, Prog. Theor. Phys. 36 (1966) 901

[11] M. Lübcke, S.M. Nasir, A.J. Niemi and K. Torokoff, Phys. Lett. B534 (2002) 195; L. Faddeev and A.J. Niemi, Phys. Rev. Lett. 85 (2000) 3416

[12] A.J. Niemi, K. Palo and S. Virtanen, Phys. Rev. D61 (2000) 085020; R.S. Ward, Phys. Rev. D66 (2002) 041701

[13] L.D. Faddeev and A.J. Niemi, Nature 387 (1997) 58

[14] E. Babaev and A.J. Niemi, to appear

[15] For a review, see A. Lerda, Anyons, Quantum Mechanics of Particles with Fractional Statistics (Springer-Verlag, Berlin 1992)

[16] L. Faddeev, A.J. Niemi and O. Viro (unpublished)

[17] L. Freyhult, Int. J. Mod. Phys. A17 (2002) 3681 